\title{
Biodegradation of phenol by pleurotus laccase in the directcurrent electric field.
}

\author{
Dajun Ren*, Xiaoyong He, Chunxing Wang, Shuqing Zhang \\ Wuhan University of Science and Technology, Wuhan, PR China
}

\begin{abstract}
In this article, the effect of direct current electric field on the phenol biodegradation by laccase was studied. The results showed that the DC electric field could enhance the biodegradation activity of laccase. Specifically, the rate of phenol biodegradation when catalyzed by laccase was significantly higher in the presence of the DC electric field than in its absence. In the presence of the DC electric field, the biodegradation rate of phenol which catalyzed by laccase was $98.6 \%$, and the phenol concentration in the residual solution was below $0.7 \mathrm{mg} / \mathrm{L}$ after $96 \mathrm{~h}$. In the absence of the electric field and with laccase, the biodegradation rate of phenol laccase was $81.7 \%$, and the phenol concentration in the residual solution was $9-10 \mathrm{mg} / \mathrm{L}$ after $96 \mathrm{~h}$. Therefore, the combination of DC electric field and laccase is likely to create a stable, simple and environmentally friendly biodegradation system.
\end{abstract}

Keywords: Biodegradation, Laccase, Direct current electric field, Phenol. Accepted on June 15, 2017

\section{Introduction}

Belonging to a family of blue oxidase enzymes, laccase is a copper-containing enzyme mainly produced by white rot fungi [1]. Laccase exhibits catalyzing capabilities in the biodegradation of various aromatic and phenolic compounds, the only product of which is water [2]. Hence, laccase is considered an environmentally friendly fermenting agent, and its role in biodegradation catalysis has been extensively studied [3]. Previously, the effects of electric fields on white rot fungi and laccase, as well as the effects of electric fields on the biodegradation of phenolic compounds were studied [4]. Nevertheless, few studies have reported the effects of electric fields on phenol biodegradation catalyzed by laccase. In this paper, the influence of DC electric field on the biodegradation of phenol by laccase was studied. On the basis of this, the effect of electric field on the biodegradability of laccase was discussed with a view to provide a stable, simple and environmentally friendly biodegradation system.

\section{Materials and Methods}

The laccase was obtained from Sigma Aldrich. Phenol was obtained from Kaitong Chemicals, China. All other chemicals were domestic analytical reagents.

Figure 1 shows the schematic view of the apparatus used for experiment. The volume of square grooves is both around 120 $\mathrm{mL}$. One of square grooves is used for direct current

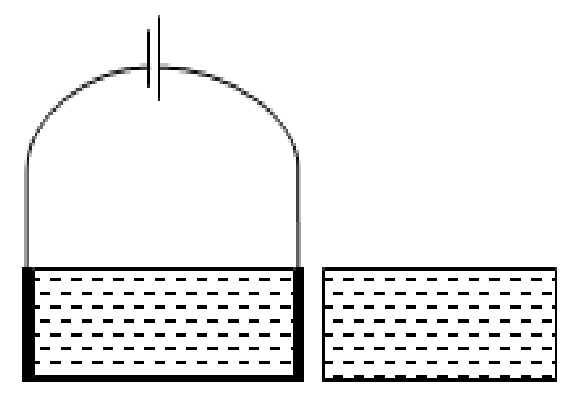

Figure 1. The apparatus used for experiment.

experiment with the graphite electrode placed at the end of it. The voltage output by DC regulated power supply to form uniform direct current electric field. The other grooves have no graphite electrode.

\section{Results and Discussion}

As shown in Figure 2, phenol degradation was observed in the control group owing to the spontaneous oxidation of phenol, while phenol degradation in the experiment with DC electric field was drastically accelerated. Furthermore, the degradation rate increased as the experiment time increased. Nevertheless, the DC electric field without laccase had a limited effect on phenol degradation, such that the overall phenol degradation rate in a prolonged period did not exceed $55 \%$. 


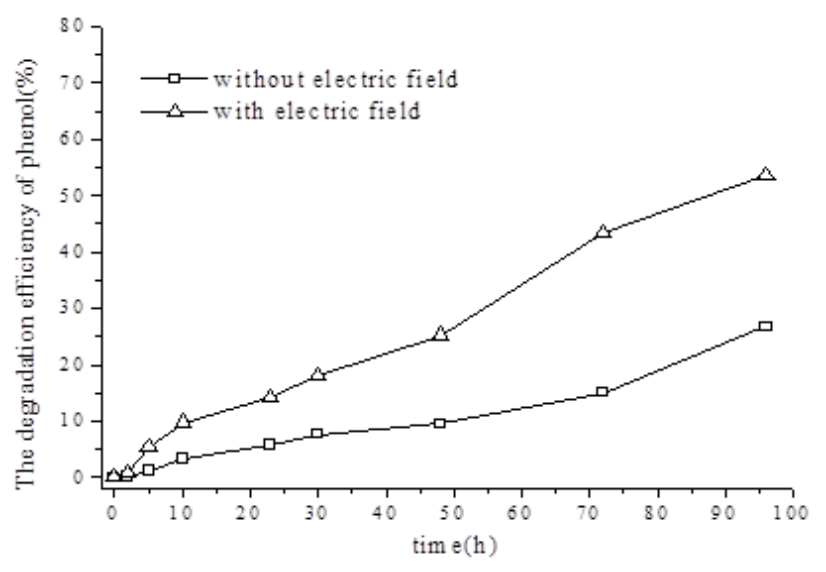

Figure 2. Degradation rate of phenol with no laccase.

An electric field can exert sophisticated effects on the reactions involved in phenol degradation. For example, the field affects both the spontaneous oxidation of phenol and catalysis by free radicals. In spontaneous oxidation, electron transfer occurs in phenol molecules because of their adsorption on the anode, leading to phenol degradation. In catalysis by free radicals, strongly oxidative free radicals such as $\cdot \mathrm{OH}$ are generated in the anode area in the presence of the DC electric field. These highly active radicals react with phenol molecules, resulting in low-toxicity intermediates, which eventually turn into $\mathrm{CO}_{2}$ and $\mathrm{H}_{2} \mathrm{O}$ through the breaking of chemical bonds and electron transfer.

Owing to the hydroxyl group in its structure, phenol exhibits weak acidity. However, it has been demonstrated that laccase exhibits higher catalytic activity in acidic environments [4]. This property makes laccase a good candidate for promoting the catalytic degradation of phenol. As shown in Figure 3, in the presence of a DC electric field, the phenol degradation rate was $98.6 \%$ after $96 \mathrm{~h}$, and the phenol concentration in the residual solution fell below $0.7 \mathrm{mg} / \mathrm{L}$. On the other hand, in the absence of the electric field, the overall phenol degradation rate was $81.7 \%$ after $96 \mathrm{~h}$, and the phenol concentration in the residual solution was $9-10 \mathrm{mg} / \mathrm{L}$. Phenol degradation catalyzed by laccase occurred at a higher rate of efficiency in the presence of the electric field than that in its absence, and this difference increased as the experiment time period increased. In the presence of a DC electric field, the degradation of phenol is accelerated by the combination of the electric field's degradation effect, the laccase's degradation effect, and the interactions between the electric field and the laccase. According to the results shown in Figures 2 and 3, laccase produces the dominant degradation effect.

Enzymatic reaction can degrade phenolic compounds through oxidation, oxidative coupling, addition, polymerization, ring breaking, or rearrangement [5]. Electrochemical effects positively impact the catalytic degradation of phenolic compounds. As shown in Figure 3, the increment of laccasecatalyzed phenol degradation in the presence of an electric field increased with the experiment time. On one hand, a DC electric field not only improves the stability of laccase and modulates processes with negative effects like the deactivation of laccase, but also exerts a direct effect on phenol degradation. On the other hand, electron transfer during phenol degradation affects the electric field.

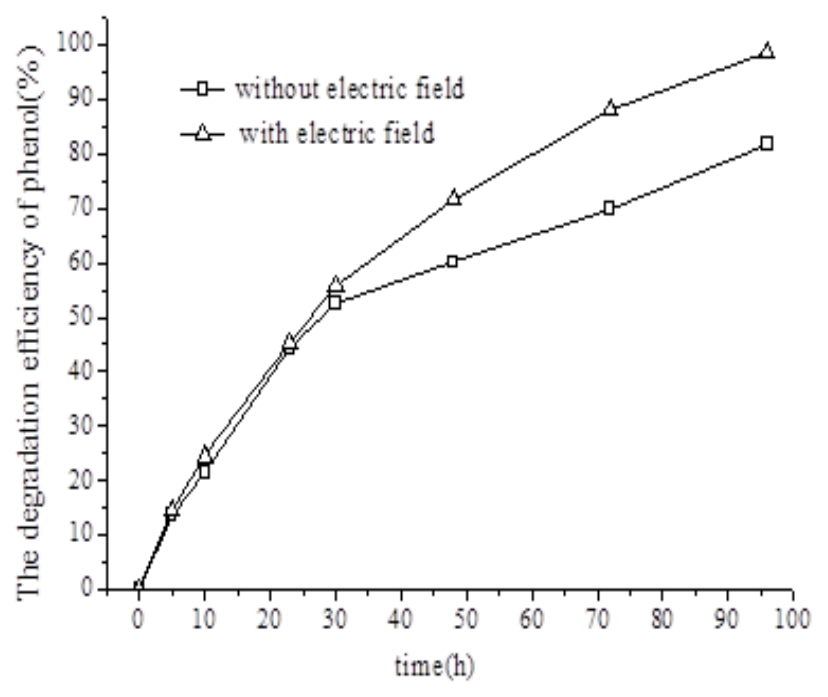

Figure 3. Degradation rate of phenol with laccase.

Laccase molecular charges are affected by its isoelectric point and the $\mathrm{pH}$ value of the solution. The charged laccase molecules, negatively-charged (due to ionization) phenol molecules, and free radicals generated in the presence of the electric field interact with each other to form various catalytic units. During prolonged experiment time, the chances that these molecules and radicals will interact with each other are relatively high. Furthermore, oxidative coupling, polymerization, ring breaking, and rearrangement of phenol radicals increase, and the catalytic efficiency is enhanced. Therefore, the degradation rate increases with the experiment time.

\section{Conclusions}

The electrochemical oxidation is a new method to degrade phenols, but this method produces only a limited increase in the rate of waste degradation. Therefore, this method should be used in conjunction with other techniques to achieve maximum efficiency in wastewater treatment. This experiment studied the interaction between DC electric field and laccase in degradation of phenol. The degradation efficiency of phenol significantly improved in the presence of the electric field, and the improvement was further amplified by prolonging the experiment time. Additionally, catalysis by laccase created the dominant effect in phenol degradation in the presence of both laccase and the DC electric field.

\section{Acknowledgements}

This work was supported by the National Natural Science Foundation of China (Grant No 41571306 and 41201306) and State Key Laboratory of Biogeology and Environmental Geology (Grant No GBL21507). 


\section{References}

1. Kolb M, Sieber V, Amann M. Removal of monomer delignification products by laccase from Trametes versicolor. Bioresource Technol 2012; 104: 298-304.

2. Ren D, Bao X, Wu G. Study on transformation of indole by laccase in the presence of phenol and ABTS. Fresenius Environ Bullet 2013; 22: 2937-2943.

3. Ren D, Zhou S, Li Q. Enhanced electrokinetic remediation of quinoline - contaminated soils. Toxicol Environ Chem 2016; 98: 585-600.

4. Wang C, Zhang H, Ren D. Effect of Direct-Current Electric Field on Catalytic Activity and the Concentration of Laccase. Indian J Microbiol 2015; 55: 1-7.
5. Oturan AM, Brillas E. Electrochemical advanced oxidation processes (EAOPs) for environmental applications. Portugaliae Electrochimca Acta 2007; 25: 1-18.

\section{*Correspondence to}

Dajun Ren

Wuhan University of Science and Technology

PR China 